\title{
ELECTRO-DISTANCE METER HEIGHT ACCURACY DETERMINA WITH RESPECT TO ENGINEERING LEVELLING
}

samir eisa
Abstract
The Electro-Distance- Meter instrument ( E.D.M.) is considered an important tool for the surveying purposes. The main aim of this article
study the theoretical analysis for the elevation difference ( Ah ) accuracy and field applications to reach a satisfied level differences between points, by modeling and simula standard deviation (a ) for (Ah ) values using E.D.M. instruments. 